\title{
On Fleck quotients
}

\author{
by \\ Zhi-Wei Sun (Nanjing) and DAQING WAN (Irvine, CA)
}

1. Introduction and main results. Let $m \in \mathbb{Z}^{+}=\{1,2, \ldots\}, n \in$ $\mathbb{N}=\{0,1, \ldots\}$ and $r \in \mathbb{Z}$, and define

$$
C_{m}(n, r)=\sum_{k \equiv r(\bmod m)}\left(\begin{array}{l}
n \\
k
\end{array}\right)(-1)^{k} .
$$

This sum has been studied by various authors and many applications have been found (cf. [S02] and the references therein). The following well-known observation is fundamental:

$$
m C_{m}(n, r)=\sum_{k=0}^{n}\left(\begin{array}{l}
n \\
k
\end{array}\right)(-1)^{k} \sum_{\gamma^{m}=1} \gamma^{k-r}=\sum_{\gamma^{m}=1} \gamma^{-r}(1-\gamma)^{n} .
$$

Note that

$$
C_{m}(n+1, r)=C_{m}(n, r)-C_{m}(n, r-1)
$$

since $x^{-r}(1-x)^{n+1}=x^{-r}(1-x)^{n}-x^{-r+1}(1-x)^{n}$.

Let $p$ be a prime, and let $n \in \mathbb{N}$ and $r \in \mathbb{Z}$. In 1913 A. Fleck (cf. [D, p. 274]) showed that

$$
\operatorname{ord}_{p}\left(C_{p}(n, r)\right) \geq\left\lfloor\frac{n-1}{p-1}\right\rfloor,
$$

where $\operatorname{ord}_{p}(\alpha)$ denotes the $p$-adic order of a $p$-adic number $\alpha$, and $\lfloor\cdot\rfloor$ is the well-known floor function. Fleck's result is fundamental in the recent investigation of the $\psi$-operator related to Fontaine's theory, Iwasawa's theory, and $p$-adic Langlands correspondence (cf. [Co], [SW] and [W]); it also plays an indispensable role in Davis and Sun's study of homotopy exponents of special unitary groups (cf. [DS] and [SD]). In this paper we are interested

2000 Mathematics Subject Classification: Primary 11B65; Secondary 05A10, 11A07, 11B68, 11B73, 11L05, 11S99.

The first author is supported by the National Science Fund for Distinguished Young Scholars (no. 10425103) in China. The second author is partially supported by NSF. 
in the Fleck quotient

$$
F_{p}(n, r):=(-p)^{-\lfloor(n-1) /(p-1)\rfloor} C_{p}(n, r)+\llbracket n=0 \rrbracket .
$$

(Throughout this paper, for an assertion $A$ we let $\llbracket A \rrbracket$ take the value 1 or 0 according as $A$ holds or not.)

For $a \in \mathbb{Z}$ and $m \in \mathbb{Z}^{+}$, we use $\{a\}_{m}$ to denote the least nonnegative residue of $a \bmod m$ (thus $\{a\}_{m} / m$ is the fractional part $\{a / m\}$ of $a / m$ ). For a prime $p$ and an integer $a$, we define $q_{p}(a)=\left(a^{p-1}-1\right) / p$, which is an integer if $a \not \equiv 0(\bmod p)$.

By a number-theoretic approach related to Gauss sums, we establish the following explicit result.

TheOREm 1.1. Let $p$ be a prime, and let $n \in \mathbb{N}$ and $r \in \mathbb{Z}$. Set $n_{0}=\{n\}_{p}$ and $n_{1}=\left\{n_{0}-n\right\}_{p-1}=\{-\lfloor n / p\rfloor\}_{p-1}$. If $n_{0} \leq n_{1}$, then

$$
F_{p}(n, r) \equiv \frac{(-1)^{n_{1}}}{n_{1} !} \sum_{k=0}^{n_{0}}\left(\begin{array}{c}
n_{0} \\
k
\end{array}\right)(-1)^{k}(k-r)^{n_{1}}(\bmod p) \text {. }
$$

If $n_{0}>n_{1}=0$, then

$$
F_{p}(n, r) \equiv(-1)^{\{r\}_{p}}\left(\begin{array}{c}
n_{0} \\
\{r\}_{p}
\end{array}\right)(\bmod p) .
$$

If $n_{0}>n_{1}>0$, then

$$
F_{p}(n, r) \equiv \frac{(-1)^{n_{1}-1}}{\left(n_{1}-1\right) !} \sum_{k=0}^{n_{0}}\left(\begin{array}{c}
n_{0} \\
k
\end{array}\right)(-1)^{k}(k-r)^{n_{1}} q_{p}(k-r)(\bmod p) .
$$

Corollary 1.1. Let $p$ be a prime and let $n \in \mathbb{N}$ and $r \in \mathbb{Z}$. Then

$$
F_{p}(p n, r) \equiv \frac{r^{n^{*}}}{n^{* !}}(\bmod p)
$$

where $n^{*}=\{-n\}_{p-1}$. Consequently,

$$
\begin{aligned}
& F_{p}\left(p \frac{p-1}{2}, r\right) \\
& \quad \equiv \begin{cases}(-1)^{(h(-p)+1) / 2}\left(\frac{r}{p}\right)(\bmod p) & \text { if } p \neq 3 \text { and } 4 \mid p+1, \\
(-1)^{(h(p)-1) / 2}\left(\frac{r}{p}\right) \frac{v}{2}(\bmod p) & \text { if } 4 \mid p-1,\end{cases}
\end{aligned}
$$

where $(\dot{p})$ is the Legendre symbol, and $h(-p)$ and $h(p)$ are the class numbers of the quadratic fields $\mathbb{Q}(\sqrt{-p})$ and $\mathbb{Q}(\sqrt{p})$ respectively, and for $p \equiv 1$ $(\bmod 4)$ we write the fundamental unit of $\mathbb{Q}(\sqrt{p})$ in the form $(v+u \sqrt{p}) / 2$ with $u, v \in \mathbb{Z}$ and $u \equiv v(\bmod 2)$. 
Proof. Note that $\{p n\}_{p}=0$. By Theorem 1.1,

$$
F_{p}(p n, r) \equiv \frac{(-1)^{n^{*}}}{n^{*} !} \sum_{k=0}^{0}\left(\begin{array}{l}
0 \\
k
\end{array}\right)(-1)^{k}(k-r)^{n^{*}}=\frac{r^{n^{*}}}{n^{*} !}(\bmod p) .
$$

When $p \neq 2$ and $n=(p-1) / 2$, we have $n^{*}=(p-1) / 2$ and hence

$$
\begin{aligned}
F_{p}\left(p \frac{p-1}{2}, r\right) & \equiv r^{(p-1) / 2}(-1)^{(p-1) / 2} \frac{((p-1) / 2) !}{\prod_{k=1}^{(p-1) / 2} k(p-k)} \\
& \equiv\left(\frac{r}{p}\right)(-1)^{(p-1) / 2} \frac{((p-1) / 2) !}{(p-1) !} \quad \text { (by Euler's criterion) } \\
& \equiv(-1)^{(p+1) / 2}\left(\frac{r}{p}\right) \frac{p-1}{2} !(\bmod p) \quad \text { (by Wilson's theorem). }
\end{aligned}
$$

If $p>3$ and $p \equiv 3(\bmod 4)$, then

$$
\frac{p-1}{2} ! \equiv(-1)^{(h(-p)+1) / 2}(\bmod p)
$$

by a result of L. J. Mordell $[\mathrm{M}]$. If $p \equiv 1(\bmod 4)$ and $\varepsilon_{p}=(v+u \sqrt{p}) / 2>1$ is the fundamental unit of $\mathbb{Q}(\sqrt{p})$ with $u, v \in \mathbb{Z}$ and $u \equiv v(\bmod 2)$, then by S. Chowla $[\mathrm{C}]$ we have

$$
\frac{p-1}{2} ! \equiv(-1)^{(h(p)+1) / 2} \frac{v}{2}(\bmod p) .
$$

Combining the above we immediately obtain (1.6).

REMARK. Let $n$ be a positive integer and $p>2 n+1$ be a prime. By the first part of Corollary 1.1 in the case $r=0$, we have

$$
\left(\begin{array}{c}
2 p n \\
p n
\end{array}\right)(-1)^{n}+2 \sum_{k=0}^{n-1}\left(\begin{array}{c}
2 p n \\
p k
\end{array}\right)(-1)^{k}=\sum_{k=0}^{2 n}\left(\begin{array}{c}
2 p n \\
p k
\end{array}\right)(-1)^{p k} \equiv 0\left(\bmod p^{2 n+1}\right)
$$

and hence

$$
\left(\begin{array}{c}
2 p n-1 \\
p n-1
\end{array}\right)=\frac{1}{2}\left(\begin{array}{c}
2 p n \\
p n
\end{array}\right) \equiv \sum_{k=0}^{n-1}(-1)^{n-1-k}\left(\begin{array}{c}
2 p n \\
p k
\end{array}\right)\left(\bmod p^{2 n+1}\right) .
$$

When $n=1$ and $p>3$, this gives the Wolstenholme congruence

$$
\frac{1}{2}\left(\begin{array}{c}
2 p \\
p
\end{array}\right)=\left(\begin{array}{c}
2 p-1 \\
p-1
\end{array}\right) \equiv 1\left(\bmod p^{3}\right) .
$$

When $n=2$ and $p>5$, (1.7) yields the following new congruence:

$$
\left(\begin{array}{c}
4 p-1 \\
2 p-1
\end{array}\right)=\frac{1}{2}\left(\begin{array}{c}
4 p \\
2 p
\end{array}\right) \equiv\left(\begin{array}{c}
4 p \\
p
\end{array}\right)-1\left(\bmod p^{5}\right) .
$$

Our second approach to Fleck quotients is of combinatorial nature. It involves Stirling numbers of the second kind as well as higher-order Bernoulli polynomials. 
Let $n \in \mathbb{N}$. The Stirling numbers $S(n, k)(k \in \mathbb{N})$ of the second kind are given by

$$
x^{n}=\sum_{k \in \mathbb{N}} S(n, k)(x)_{k},
$$

where

$$
(x)_{0}=1 \quad \text { and } \quad(x)_{k}=x(x-1) \cdots(x-k+1) \quad \text { for } k=1,2, \ldots .
$$

Clearly, $S(n, n)=1$, and $S(n, k)=0$ if $k>n$. When $n+k>0, S(n, k)$ is actually the number of ways to partition a set of cardinality $n$ into $k$ nonempty subsets. Here is an explicit formula (cf. [LW, p. 126]) for Stirling numbers of the second kind:

$$
S(n, k)=\frac{1}{k !} \sum_{j=0}^{k}\left(\begin{array}{l}
k \\
j
\end{array}\right)(-1)^{k-j} j^{n} .
$$

As $S(i, k)=0$ for all those $i \in \mathbb{N}$ with $i<k$, we have Euler's identity

$$
\sum_{j=0}^{k}\left(\begin{array}{c}
k \\
j
\end{array}\right)(-1)^{j} P(j)=0
$$

where $P(x)$ is any polynomial with $\operatorname{deg} P<k$ having complex number coefficients. It is known (cf. [LW, p. 126]) that

$$
\sum_{n=k}^{\infty} S(n, k) \frac{x^{n}}{n !}=\frac{\left(e^{x}-1\right)^{k}}{k !}
$$

in other words,

$$
\left(e^{x}-1\right)^{k}=\sum_{n=k}^{\infty} \bar{S}(n, k) x^{n} \quad \text { with } \quad \bar{S}(n, k)=\frac{k !}{n !} S(n, k) .
$$

For $m=0,1, \ldots$, the $m$ th order Bernoulli polynomials $B_{n}^{(m)}(t)(n \in \mathbb{N})$ are defined by

$$
\frac{x^{m} e^{t x}}{\left(e^{x}-1\right)^{m}}=\sum_{n=0}^{\infty} B_{n}^{(m)}(t) \frac{x^{n}}{n !},
$$

and those $B_{n}^{(m)}=B_{n}^{(m)}(0)$ are called the mth order Bernoulli numbers. The usual Bernoulli polynomials and numbers are $B_{n}(t)=B_{n}^{(1)}(t)$ and $B_{n}=B_{n}(0)=B_{n}^{(1)}$ respectively. (It is well known that $B_{0}=1, B_{1}=-1 / 2$ and $B_{2 k+1}=0$ for $k=1,2, \ldots$; the reader may consult [IR, pp. 228-248] for the basic properties of Bernoulli numbers.) For a formal power series $f(x)=\sum_{n=0}^{\infty} a_{n} x^{n}$, we use $\left[x^{n}\right] f(x)$ to denote the coefficient $a_{n}$ of the monomial $x^{n}$ in $f(x)$. Thus 


$$
\begin{aligned}
B_{n}^{(m)}(t) & =\left[x^{n}\right] n !\left(\frac{x}{e^{x}-1}\right)^{m} e^{t x} \\
& =\left[x^{n}\right] n ! \sum_{k=0}^{\infty} B_{k}^{(m)} \frac{x^{k}}{k !} \sum_{j=0}^{\infty} \frac{(t x)^{j}}{j !}=\sum_{k=0}^{n}\left(\begin{array}{l}
n \\
k
\end{array}\right) B_{k}^{(m)} t^{n-k} .
\end{aligned}
$$

It is also easy to verify that $B_{n}^{(m)}(m-t)=(-1)^{n} B_{n}^{(m)}(t)$, and

$$
\frac{B_{n}^{(m)}(t)}{n !}=\sum_{k_{0}+\cdots+k_{m-1}=n} \frac{B_{k_{0}}(t)}{k_{0} !} \prod_{0<i<m} \frac{B_{k_{i}}}{k_{i} !} \quad \text { provided } m>0 .
$$

If $0 \leq n<p-1$, then $B_{0}, \ldots, B_{n}$ are $p$-adic integers by the von StaudtClausen theorem (cf. [IR, p. 233]) or the recurrence $\sum_{k=0}^{l}\left(\begin{array}{c}l+1 \\ k\end{array}\right) B_{k}=0$ $(l=1,2, \ldots)$, therefore $B_{n}^{(m)}(t) \in \mathbb{Z}_{p}[t]$ where $\mathbb{Z}_{p}$ is the ring of $p$-adic integers.

Our discovery of the next theorem was actually motivated by Theorem 1.1.

TheOREM 1.2. Let $p$ be a prime, and let $n \in \mathbb{N}$ and $r \in \mathbb{Z}$. Set $n^{*}=$ $\{-n\}_{p-1}$. For any integer $m \equiv n(\bmod p)$, if $m \geq 0$ then $(-1)^{n} F_{p}(n, r)$ is congruent to

$$
\begin{aligned}
\sum_{k=0}^{n^{*}} \bar{S}\left(n^{*}-k+m, m\right) \frac{(-r)^{k}}{k !} & =\sum_{k=0}^{n^{*}} \bar{S}\left(m+n^{*}, m+k\right)\left(\begin{array}{c}
-r \\
k
\end{array}\right) \\
& =\sum_{k=0}^{m}\left(\begin{array}{c}
m \\
k
\end{array}\right)(-1)^{m-k} \frac{(k-r)^{m+n^{*}}}{\left(m+n^{*}\right) !}
\end{aligned}
$$

modulo $p$; if $m \leq 0$ then we have

(1.10) $\quad F_{p}(n, r)$

$$
\equiv \frac{(-1)^{n^{*}}}{n^{*} !} B_{n^{*}}^{(-m)}(-r) \equiv-\left(p-1-n^{*}\right) ! B_{n^{*}}^{(-m)}(-r)(\bmod p) .
$$

The following consequence determines $B_{n}^{(m)}(a)$ modulo a prime $p$ for $m \in\{1, \ldots, p\}, n \in\{0, \ldots, p-2\}$ and $a \in \mathbb{Z}$.

Corollary 1.2. Let $p$ be a prime and $r \in \mathbb{Z}$. Let $n_{0} \in\{0, \ldots, p-1\}$ and $n_{1} \in\{0, \ldots, p-2\}$. If $n_{0} \leq n_{1}$, then

$$
B_{n_{1}-n_{0}}^{\left(p-n_{0}\right)}(-r) \equiv \frac{1}{\left(n_{1}\right)_{n_{0}}} \sum_{k=0}^{n_{0}}\left(\begin{array}{c}
n_{0} \\
k
\end{array}\right)(-1)^{n_{0}-k}(k-r)^{n_{1}}(\bmod p) .
$$

If $n_{0}>n_{1}=0$, then

$$
B_{p-n_{0}+n_{1}-1}^{\left(p-n_{0}\right)}(-r) \equiv \frac{(-1)^{\{r\}_{p}-1}}{n_{0} !}\left(\begin{array}{c}
n_{0} \\
\{r\}_{p}
\end{array}\right)(\bmod p)
$$


If $n_{0}>n_{1}>0$, then

$$
\begin{aligned}
& B_{p-n_{0}+n_{1}-1}^{\left(p-n_{0}\right)}(-r) \\
& \equiv \frac{(-1)^{n_{1}}}{\left(n_{0}-n_{1}\right) !\left(n_{1}-1\right) !} \sum_{k=0}^{n_{0}}\left(\begin{array}{c}
n_{0} \\
k
\end{array}\right)(-1)^{k}(k-r)^{n_{1}} q_{p}(k-r)(\bmod p) .
\end{aligned}
$$

Proof. Let $n$ be a nonnegative integer with $n \equiv n_{0}-p n_{1}(\bmod p(p-1))$. Applying (1.10) with $m=n_{0}-p$ we obtain

$$
F_{p}(n, r) \equiv \frac{(-1)^{n^{*}}}{n^{*} !} B_{n^{*}}^{\left(p-n_{0}\right)}(-r) \equiv-\left(p-1-n^{*}\right) ! B_{n^{*}}^{\left(p-n_{0}\right)}(-r)(\bmod p),
$$

where $n^{*}=\{-n\}_{p-1}$.

If $n_{0} \leq n_{1}$, then $n^{*}=n_{1}-n_{0}$ and hence

$$
B_{n_{1}-n_{0}}^{\left(p-n_{0}\right)}(-r) \equiv(-1)^{n_{1}-n_{0}}\left(n_{1}-n_{0}\right) ! F_{p}(n, r)(\bmod p),
$$

which implies (1.11) with the help of (1.2).

Now we consider the case $n_{0}>n_{1}$. Clearly $n^{*}=n_{1}-n_{0}+p-1$ and $p-1-n^{*}=n_{0}-n_{1}$. Therefore

$$
F_{p}(n, r) \equiv-\left(n_{0}-n_{1}\right) ! B_{n_{1}-n_{0}+p-1}^{\left(p-n_{0}\right)}(-r)(\bmod p) .
$$

The case $n_{1}=0$ of this, together with (1.3), yields (1.12). When $n_{1}>0$, combining the last congruence with (1.4) we obtain (1.13).

Corollary 1.3. Let $p$ be a prime and let $n \in \mathbb{Z}^{+}$. Then $\operatorname{ord}_{p}\left(C_{p}(n, r)\right)=$ $\lfloor(n-1) /(p-1)\rfloor$ for at least $p-n^{*} \geq 2$ values of $r \in\{0, \ldots, p-1\}$, where $n^{*}=\{-n\}_{p-1}$.

Proof. For any $r \in \mathbb{Z}, \operatorname{ord}_{p}\left(C_{p}(n, r)\right)=\lfloor(n-1) /(p-1)\rfloor$ if and only if $F_{p}(n, r) \not \equiv 0(\bmod p)$. By Theorem 1.2 ,

$$
F_{p}(n, r) \equiv \frac{(-1)^{n^{*}}}{n^{*} !} B_{n^{*}}^{\left(p-\{n\}_{p}\right)}(-r)(\bmod p) \quad \text { for all } r=0, \ldots, p-1 .
$$

Recall that $B_{n^{*}}^{\left(p-\{n\}_{p}\right)}(x) \in \mathbb{Z}_{p}[x]$ is monic and of degree $n^{*}$. Also, a polynomial of degree $n^{*}$ over the field $\mathbb{Z} / p \mathbb{Z}$ cannot have more than $n^{*}$ distinct zeroes in the field (cf. [IR, p. 39]). So the congruence equation $F_{p}(n, r) \equiv 0$ $(\bmod p)$ has at most $n^{*}$ solutions with $r \in\{0, \ldots, p-1\}$. This yields the desired result. Then

$$
(-1)^{n} F_{p}(n, 0) \equiv \bar{S}\left(n^{*}+\{n\}_{p},\{n\}_{p}\right) \equiv \frac{B_{n^{*}}^{(m)}}{n^{*} !}(\bmod p),
$$

where $m$ is any nonnegative integer with $m+n \equiv 0(\bmod p)$. Also, 


$$
\begin{aligned}
& (-1)^{n} F_{p}(p n+p-1, r) \\
& \quad \equiv \frac{B_{n^{*}}(-r)}{n^{*} !} \equiv-\left(p-1-n^{*}\right) ! B_{n^{*}}(r+1)(\bmod p)
\end{aligned}
$$

for all $r \in \mathbb{Z}$, and in particular

$$
\left(\begin{array}{c}
2 p-1 \\
p+r
\end{array}\right)+(-1)^{p}\left(\begin{array}{c}
2 p-1 \\
r
\end{array}\right) \equiv(-1)^{r} p^{2} B_{p-2}(-r)\left(\bmod p^{3}\right)
$$

for every $r=0, \ldots, p-1$.

Proof. Applying Theorem 1.2 with $r=0$ we immediately get (1.14).

As $p n+p-1 \equiv-1(\bmod p)$ and $n^{*}=\{-(p n+p-1)\}_{p-1}$, by the second part of Theorem 1.2 and the identity $(-1)^{n^{*}} B_{n^{*}}(x)=B_{n^{*}}(1-x)$, whenever $r \in \mathbb{Z}$ we have

$$
\begin{aligned}
(-1)^{n^{*}} F_{p}(p n+p-1, r) & \equiv \frac{B_{n^{*}}(-r)}{n^{*} !} \equiv(-1)^{n^{*}+1}\left(p-1-n^{*}\right) ! B_{n^{*}}(-r) \\
& \equiv-\left(p-1-n^{*}\right) ! B_{n^{*}}(r+1)(\bmod p)
\end{aligned}
$$

and hence (1.15) holds.

Now let $r \in\{0, \ldots, p-1\}$. By (1.15) in the case $n=1$,

$$
-F_{p}(2 p-1, r) \equiv-(p-1-(p-2)) ! B_{p-2}(r+1)(\bmod p)
$$

and hence

$$
F_{p}(2 p-1, r) \equiv B_{p-2}(1-(-r))=(-1)^{p-2} B_{p-2}(-r)(\bmod p),
$$

which is equivalent to (1.16).

Let $p$ be an odd prime, and let $h_{p}$ and $h_{p}^{+}$denote the class numbers of the cyclotomic field $\mathbb{Q}\left(\zeta_{p}\right)$ and its maximal real subfield $\mathbb{Q}\left(\zeta_{p}+\zeta_{p}^{-1}\right)$ respectively, where $\zeta_{p}$ is a primitive $p$ th root of unity in the complex field $\mathbb{C}$. It is well known that $h_{p}^{-}=h_{p} / h_{p}^{+}$is an integer. If $p$ divides none of the numerators of the Bernoulli numbers $B_{0}, B_{2}, \ldots, B_{p-3} \in \mathbb{Z}_{p}$, then $p$ is said to be a regular prime. In $1850 \mathrm{E}$. Kummer proved that

$$
\begin{aligned}
p \nmid h_{p} & \Leftrightarrow p \nmid h_{p}^{-} \Leftrightarrow p \text { is regular } \\
& \Rightarrow x^{p}+y^{p}=z^{p} \text { has no integer solution with } x y z \neq 0 .
\end{aligned}
$$

Furthermore,

$$
h_{p}^{-} \equiv \prod_{0<n \leq(p-3) / 2}\left(-\frac{B_{2 n}}{4 n}\right)(\bmod p)
$$

by the proof of Theorem 5.16 in [Wa, p. 62]. 
Corollary 1.5. Let $p$ be a prime.

(i) For every $n=2, \ldots, p$ we have

$$
\sum_{k=1}^{n}(-1)^{p k-1}\left(\begin{array}{c}
p n-1 \\
p k-1
\end{array}\right) \equiv(n-1) ! B_{p-n} p^{n}\left(\bmod p^{n+1}\right) .
$$

(ii) Suppose that $p>3$. Then $p$ does not divide the class number $h_{p}$ of the pth cyclotomic field $\mathbb{Q}\left(\zeta_{p}\right)$ if and only if

$$
\operatorname{ord}_{p}\left(\sum_{k=1}^{n}(-1)^{k}\left(\begin{array}{l}
p n-1 \\
p k-1
\end{array}\right)\right)=n \quad \text { for all } n=3,5, \ldots, p-2 .
$$

Also,

$$
\begin{aligned}
& \sum_{k=1}^{(p-1) / 2}(-1)^{k-1}\left(\begin{array}{c}
p(p-1) / 2-1 \\
p k-1
\end{array}\right) \\
& \quad \equiv \llbracket 4 \mid p+1 \rrbracket(-1)^{(h(-p)+1) / 2} h(-p) p^{(p-1) / 2}\left(\bmod p^{(p+1) / 2}\right),
\end{aligned}
$$

where $h(-p)$ is the class number of the imaginary quadratic field $\mathbb{Q}(\sqrt{-p})$.

Proof. (i) Let $n \in\{2, \ldots, p\}$. Then $\lfloor(p n-1-1) /(p-1)\rfloor=n$ and hence $F_{p}(p n-1,-1)=(-p)^{-n} C_{p}(p n-1,-1)=(-p)^{-n} \sum_{k=1}^{n}\left(\begin{array}{l}p n-1 \\ p k-1\end{array}\right)(-1)^{p k-1}$.

By Corollary $1.4,(-1)^{n} F_{p}(p n-1,-1)$ is congruent to

$$
\left(p-1-\{-(n-1)\}_{p-1}\right) ! B_{\{-(n-1)\}_{p-1}}(-1+1)=(n-1) ! B_{p-n}
$$

modulo $p$. Therefore (1.17) holds.

(ii) In view of part (i),

$$
\begin{gathered}
\operatorname{ord}_{p}\left(\sum_{k=1}^{n}(-1)^{k}\left(\begin{array}{c}
p n-1 \\
p k-1
\end{array}\right)\right)=n \quad \text { for } n=3,5, \ldots, p-2 \\
\Leftrightarrow B_{p-n} \not \equiv 0(\bmod p) \text { for } n=3,5, \ldots, p-2 \\
\Leftrightarrow p \text { is regular } \Leftrightarrow h_{p} \neq \equiv 0(\bmod p) .
\end{gathered}
$$

Taking $n=(p-1) / 2$ in (1.17) we get

$$
\begin{aligned}
& \sum_{k=1}^{(p-1) / 2}(-1)^{k-1}\left(\begin{array}{c}
p(p-1) / 2-1 \\
p k-1
\end{array}\right) \\
& \equiv \frac{((p-1) / 2) !}{(p-1) / 2} p^{(p-1) / 2} B_{(p+1) / 2}\left(\bmod p^{(p+1) / 2}\right) .
\end{aligned}
$$

If $p \equiv 1(\bmod 4)$, then $B_{(p+1) / 2}=0$ since $(p+1) / 2 \in\{3,5, \ldots\}$. If $p \equiv 3$ $(\bmod 4)$, then we have $h(-p) \equiv-2 B_{(p+1) / 2}(\bmod p)(\mathrm{cf} .[$ IR, p. 238]), and 
$((p-1) / 2) ! \equiv(-1)^{(h(-p)+1) / 2}(\bmod p)$ by Mordell $[\mathrm{M}]$. So $(1.18)$ follows from the above.

REMARK. Let $p$ be an odd prime. If $p \geq 5$, then (1.17) in the case $n=2$ reduces to Wolstenholme's congruence $\left(\begin{array}{c}2 p-1 \\ p-1\end{array}\right) \equiv 1\left(\bmod p^{3}\right)$ since $B_{p-2}=0$. Taking $n=3$ in (1.17) we get

$$
\left(\begin{array}{c}
3 p-1 \\
p-1
\end{array}\right)-\left(\begin{array}{c}
3 p-1 \\
2 p-1
\end{array}\right)+\left(\begin{array}{c}
3 p-1 \\
3 p-1
\end{array}\right) \equiv 2 B_{p-3} p^{3}\left(\bmod p^{4}\right) ;
$$

as $\left(\begin{array}{c}3 p-1 \\ 2 p-1\end{array}\right)=2\left(\begin{array}{c}3 p-1 \\ p-1\end{array}\right)$ this yields the congruence

$$
\left(\begin{array}{c}
3 p-1 \\
p-1
\end{array}\right) \equiv 1-2 p^{3} B_{p-3}\left(\bmod p^{4}\right) .
$$

This was first obtained by J. W. L. Glaisher (cf. [G1, p. 21] and [G2, p. 323]) who showed that

$$
\left(\begin{array}{c}
p n-1 \\
p-1
\end{array}\right) \equiv 1-\frac{n(n-1)}{3} p^{3} B_{p-3}\left(\bmod p^{4}\right) \quad \text { for } n=1,2, \ldots
$$

Corollary 1.6. Let $p$ be an odd prime, and let $n \in\{3, \ldots, p\}$ and $r \in \mathbb{Z}$. Then

$$
F_{p}(p n-2, r) \equiv-n !\left(\frac{B_{p-n+1}(-r)}{n-1}+(r+1) \frac{B_{p-n}(-r)}{n}\right)(\bmod p) .
$$

Proof. Clearly $\{-(p n-2)\}_{p-1}=p-n+1$. By Theorem 1.2, $F_{p}(p n-2, r)$ is congruent to

$$
-(p-1-(p-n+1)) ! B_{p-n+1}^{(2)}(-r)=-(n-2) ! B_{p-n+1}^{(2)}(-r)
$$

modulo $p$.

Let $m=p-n+1$. By [PS, (2.14)] or [SP, (1.12)],

$$
\begin{aligned}
\frac{(-1)^{m}}{m} & \sum_{k=0}^{m}\left(\begin{array}{c}
m \\
k
\end{array}\right) B_{k} B_{m-k}(x)-\frac{B_{m}(1-x)}{m} B_{0} \\
& =-\sum_{k=0}^{1}\left(\begin{array}{l}
1 \\
k
\end{array}\right) B_{1-k}(x) B_{m-1+k}(1-x)-B_{1} B_{m-1}(1-x) \\
& =-B_{1}(x) B_{m-1}(1-x)-B_{0}(x) B_{m}(1-x)-B_{1} B_{m-1}(1-x) \\
& =(-1)^{m}\left(\left(B_{1}(x)+B_{1}\right) B_{m-1}(x)-B_{m}(x)\right) \\
& =(-1)^{m}\left((x-1) B_{m-1}(x)-B_{m}(x)\right) .
\end{aligned}
$$


It follows that

$$
\begin{aligned}
B_{m}^{(2)}(-r) & =\sum_{k=0}^{m}\left(\begin{array}{c}
m \\
k
\end{array}\right) B_{k} B_{m-k}(-r) \\
& =(1-m) B_{m}(-r)+m(-r-1) B_{m-1}(-r) \\
& \equiv(1+n-1) B_{p-n+1}(-r)-(r+1)(-n+1) B_{p-n}(-r) \\
& \equiv n(n-1)\left(\frac{B_{p-n+1}(-r)}{n-1}+(r+1) \frac{B_{p-n}(-r)}{n}\right)(\bmod p) .
\end{aligned}
$$

Combining the above we immediately obtain (1.19).

By Theorem 1.1 or 1.2 , for any prime $p$ the Fleck quotient $F_{p}(n, r)$ (with $n \in \mathbb{N}$ and $r \in \mathbb{Z}$ ) modulo $p$ only depends on $p$ and $r$ and the remainder of $n$ modulo $p(p-1)$. This observation can be further extended as follows.

Theorem 1.3. Let $p$ be a prime, and let $a, l, n \in \mathbb{N}$ and $r \in \mathbb{Z}$. Then

$$
\sum_{k=0}^{n}\left(\begin{array}{l}
n \\
k
\end{array}\right)(-1)^{k} F_{p}\left(k p^{a}(p-1)+l, r\right) \equiv 0\left(\bmod p^{a n+\left\lceil\left(n-l^{*}\right) /(p-1)\right\rceil}\right),
$$

where $l^{*}=\{-l\}_{p-1}$ and $\lceil\cdot\rceil$ is the ceiling function.

The following consequence is somewhat similar to Kummer's congruence for Bernoulli numbers (cf. [IR, pp. 238-241]).

Corollary 1.7. Let $p$ be a prime, and let $a, l \in \mathbb{N}$ and $r \in \mathbb{Z}$. Then

$$
\begin{aligned}
F_{p}\left(p^{a}(p-1)+l, r\right) \equiv & F_{p}(l, r)\left(\bmod p^{a}\right), \\
F_{p}\left(2 p^{a}(p-1)+l, r\right) \equiv & 2 F_{p}\left(p^{a}(p-1)+l, r\right)-F_{p}(l, r)\left(\bmod p^{2 a}\right), \\
F_{p}\left(3 p^{a}(p-1)+l, r\right) \equiv & 3 F_{p}\left(2 p^{a}(p-1)+l, r\right)-3 F_{p}\left(p^{a}(p-1)+l, r\right) \\
& +F_{p}(l, r)\left(\bmod p^{3 a}\right) .
\end{aligned}
$$

Proof. Simply apply (1.20) with $n=1,2,3$.

Let $p$ be a prime, and let $a \in \mathbb{Z}^{+}$and $r \in \mathbb{Z}$. In 1977 C. S. Weisman [We] extended Fleck's result by showing that if $n \geq p^{a-1}$ then

$$
C_{p^{a}}(n, r) \equiv 0\left(\bmod p^{\left\lfloor\left(n-p^{a-1}\right) / \varphi\left(p^{a}\right)\right\rfloor}\right),
$$

where $\varphi$ is Euler's totient function. In view of this, we define the generalized Fleck quotient

$$
F_{p^{a}}(n, r)=(-p)^{-\left\lfloor\left(n-p^{a-1}\right) / \varphi\left(p^{a}\right)\right\rfloor} C_{p^{a}}(n, r)+\llbracket n<p^{a-1} \rrbracket \in \mathbb{Z} .
$$

Note that $F_{p^{a}}(n, r) \equiv 1(\bmod p)$ for $n=0, \ldots, p^{a-1}-1$. 
Theorem 1.4. Let $p$ be a prime, and let $a, n \in \mathbb{Z}^{+}$with $n \geq p^{a-1}$.

(i) For any $r \in \mathbb{Z}$ we have

$$
F_{p^{a}}(n, r) \equiv \sum_{k=0}^{d}\left(\begin{array}{c}
r+k-1 \\
k
\end{array}\right) F_{p^{a}}(n+k, 0)(\bmod p),
$$

where $d=\left\{p^{a-1}-1-n\right\}_{\varphi\left(p^{a}\right)}$ is the least nonnegative integer with $n+d \equiv p^{a-1}-1\left(\bmod \varphi\left(p^{a}\right)\right)$.

(ii) We have

$$
\left.\operatorname{ord}_{p}\left(C_{p^{a}}(n, r)\right)=\left\lfloor\frac{n-p^{a-1}}{\varphi\left(p^{a}\right)}\right\rfloor \quad \text { (i.e., } p \nmid F_{p^{a}}(n, r)\right) \text { for some } r \in \mathbb{Z} .
$$

If $n \geq 2 p^{a-1}$, then

$$
F_{p^{a}}\left(n+p^{a}(p-1), r\right) \equiv F_{p^{a}}(n, r)(\bmod p) \quad \text { for all } r \in \mathbb{Z} .
$$

In view of the first congruence in Corollary 1.7 and the last congruence in Theorem 1.4, we propose the following conjecture.

Conjecture 1.1. Let $p$ be a prime, and let $a, b, n \in \mathbb{Z}^{+}$and $r \in \mathbb{Z}$. If $n \geq 2 p^{a+b-2}$, then

$$
F_{p^{a}}\left(n+\varphi\left(p^{a+b}\right), r\right) \equiv F_{p^{a}}(n, r)\left(\bmod p^{b}\right) .
$$

Theorems 1.1, 1.2 and 1.3 will be proved in Sections 2, 3 and 4 respectively. In Section 5 we will first give a new proof of Weisman's congruence via roots of unity, and then establish Theorem 1.4.

\section{Proof of Theorem 1.1}

Lemma 2.1. Let $p$ be a prime, and let $n \in \mathbb{N}$ and $n^{*}=\{-n\}_{p-1}$. Define $G(n)=\sum_{a=1}^{p-1} a^{n} \zeta_{p}^{a}$ and $\pi=1-\zeta_{p}$, where $\zeta_{p}$ is a primitive pth root of unity in the complex field $\mathbb{C}$. Then

$$
G(n) \equiv(-1)^{n^{*}-1} \sum_{m=n^{*}}^{p-2} s\left(m, n^{*}\right) \frac{\pi^{m}}{m !}(\bmod p),
$$

where $s(m, 0), \ldots, s(m, m)$ are Stirling numbers of the first kind defined by $(x)_{m}=\sum_{k=0}^{m}(-1)^{m-k} s(m, k) x^{k}$. 
Proof. Clearly,

$$
\begin{aligned}
G(n) & =\sum_{a=1}^{p-1} a^{n}(1-\pi)^{a}=\sum_{a=1}^{p-1} a^{n} \sum_{m=0}^{a}\left(\begin{array}{c}
a \\
m
\end{array}\right)(-\pi)^{m}=\sum_{m=0}^{p-1} \frac{(-\pi)^{m}}{m !} \sum_{a=1}^{p-1} a^{n}(a)_{m} \\
& =\sum_{m=0}^{p-1} \frac{(-\pi)^{m}}{m !} \sum_{a=1}^{p-1} a^{n} \sum_{k=0}^{m}(-1)^{m-k} s(m, k) a^{k} \\
& =\sum_{m=0}^{p-1} \frac{(-\pi)^{m}}{m !} \sum_{k=0}^{m}(-1)^{m-k} s(m, k) \sum_{a=1}^{p-1} a^{n+k} .
\end{aligned}
$$

Since

$$
1+x+\cdots+x^{p-1}=\frac{x^{p}-1}{x-1}=\prod_{a=1}^{p-1}\left(x-\zeta_{p}^{a}\right)
$$

we have

$$
\frac{p}{\pi^{p-1}}=\prod_{a=1}^{p-1} \frac{1-\zeta_{p}^{a}}{\pi}=\prod_{a=1}^{p-1} \frac{1-(1-\pi)^{a}}{\pi} \equiv \prod_{a=1}^{p-1} a \equiv-1(\bmod \pi)
$$

with the help of Wilson's theorem. Note also that

$$
\sum_{a=1}^{p-1} a^{n+k} \equiv-\llbracket p-1 \mid n+k \rrbracket(\bmod p)
$$

by elementary number theory (see, e.g., [IR, pp. 235-236]). Therefore

$$
\begin{aligned}
G(n) & \equiv \sum_{m=0}^{p-2} \frac{\pi^{m}}{m !} \sum_{k=0}^{m}(-1)^{k} s(m, k)\left(-\llbracket k=n^{*} \rrbracket\right) \\
& \equiv(-1)^{n^{*}-1} \sum_{m=n^{*}}^{p-2} s\left(m, n^{*}\right) \frac{\pi^{m}}{m !}(\bmod p) .
\end{aligned}
$$

REMARK. Let $p$ be an odd prime. For each $a \in \mathbb{Z}$ let $\bar{a}=a+p \mathbb{Z} \in$ $\mathbb{F}_{p}=\mathbb{Z} / p \mathbb{Z}$. Let $\omega$ be the Teichmüller character of the multiplicative group $\mathbb{F}_{p}^{*}=\mathbb{F}_{p} \backslash\{\overline{0}\}$. For $\bar{a} \in \mathbb{F}_{p}^{*}, \omega(\bar{a})$ is just the $(p-1)$ th root of unity in the unique unramified extension of the $p$-adic field $\mathbb{Q}_{p}$ with $\omega(\bar{a}) \equiv a(\bmod p)$. (See, e.g., [Wa, p. 51].) If $\zeta_{p}$ is a primitive $p$ th root of unity in the algebraic closure of $\mathbb{Q}_{p}$, then for $n \in \mathbb{N}$ and $\pi=1-\zeta_{p}$ we have

$$
\sum_{a=1}^{p-1} a^{n} \zeta_{p}^{a} \equiv \sum_{a=1}^{p-1} \omega^{n}(\bar{a}) \zeta_{p}^{a} \equiv-\frac{(-\pi)^{n^{*}}}{n^{*} !}\left(\bmod \pi^{n^{*}+1}\right)
$$

with $n^{*}=\{-n\}_{p-1}$, by Stickelberger's congruence for Gauss' sums (cf. [BEW, pp. 344-345]). 
LEMma 2.2. Let $p$ be a prime, and let $\zeta_{p}$ be a primitive pth root of unity in $\mathbb{C}$. Let $n=p^{a} m+n_{0}>0$ with $a \in \mathbb{Z}^{+}$and $m, n_{0} \in \mathbb{N}$. Then for any $r \in \mathbb{Z}$ we have

$$
\begin{aligned}
& \pi^{-p^{a} m} C_{p}(n, r)-\llbracket p-1 \mid m \rrbracket C_{p}\left(n_{0}, r\right) \\
& \equiv \frac{G\left(p^{a} m\right)}{p} \sum_{k=0}^{n_{0}}\left(\begin{array}{c}
n_{0} \\
k
\end{array}\right)(-1)^{k}(k-r)^{p^{a} m^{*}}\left(\bmod p^{a-1} \pi^{\min \left\{n_{0}+1, p-1\right\}}\right),
\end{aligned}
$$

where $\pi=1-\zeta_{p}$ and $m^{*}=\{-m\}_{p-1}$.

Proof. Let $j \in\{1, \ldots, p-1\}$. Then

$$
\left(\frac{1-\zeta_{p}^{j}}{\pi}\right)^{m}=\left(\frac{1-(1-\pi)^{j}}{\pi}\right)^{m}=\left(\sum_{i=1}^{j}\left(\begin{array}{l}
j \\
i
\end{array}\right)(-\pi)^{i-1}\right)^{m}=j^{m}+\beta_{j} \pi,
$$

where $\beta_{j}$ is a suitable element in the ring $\overline{\mathbb{Z}}$ of algebraic integers. For $i=$ $0,1, \ldots$, if

$$
\left(\frac{1-\zeta_{p}^{j}}{\pi}\right)^{p^{i} m}=j^{p^{i} m}+p^{i} \pi \beta_{j}^{(i)}
$$

for some $\beta_{j}^{(i)} \in \overline{\mathbb{Z}}$, then

$$
\left(\frac{1-\zeta_{p}^{j}}{\pi}\right)^{p^{i+1} m}=\left(j^{p^{i} m}+p^{i} \pi \beta_{j}^{(i)}\right)^{p}=j^{p^{i+1} m}+p^{i+1} \pi \beta_{j}^{(i+1)}
$$

for some $\beta_{j}^{(i+1)} \in \overline{\mathbb{Z}}$. So

$$
\left(\frac{1-\zeta_{p}^{j}}{\pi}\right)^{p^{a} m} \equiv j^{p^{a} m}\left(\bmod p^{a} \pi\right)
$$

Observe that

$$
p C_{p}(n, r)=\sum_{j=0}^{p-1} \zeta_{p}^{-j r}\left(1-\zeta_{p}^{j}\right)^{n}=\pi^{p^{a} m} \sum_{j=1}^{p-1} \zeta_{p}^{-j r}\left(\frac{1-\zeta_{p}^{j}}{\pi}\right)^{p^{a} m}\left(1-\zeta_{p}^{j}\right)^{n_{0}} .
$$

As $\pi^{n_{0}}$ divides $\left(1-\zeta_{p}^{j}\right)^{n_{0}}$ in the ring $\overline{\mathbb{Z}}$, by the above $\pi^{-p^{a} m} p C_{p}(n, r)$ is congruent to

$$
\sum_{j=1}^{p-1} \zeta_{p}^{-j r} j^{p^{a} m} \sum_{k=0}^{n_{0}}\left(\begin{array}{c}
n_{0} \\
k
\end{array}\right)(-1)^{k} \zeta_{p}^{j k}=\sum_{k=0}^{n_{0}}\left(\begin{array}{c}
n_{0} \\
k
\end{array}\right)(-1)^{k} S_{k-r}
$$

modulo $p^{a} \pi^{n_{0}+1}$, where

$$
S_{k-r}=\sum_{j=1}^{p-1} j^{p^{a} m} \zeta_{p}^{j(k-r)}
$$


If $k \not \equiv r(\bmod p)$, then

$$
\begin{aligned}
S_{k-r} & =(k-r)^{-p^{a} m} \sum_{j=1}^{p-1}(j(k-r))^{p^{a} m} \zeta_{p}^{j(k-r)} \\
& \equiv(k-r)^{p^{a} m^{*}} \sum_{t=1}^{p-1} t^{p^{a} m} \zeta_{p}^{t}=(k-r)^{p^{a} m^{*}} G\left(p^{a} m\right)\left(\bmod p^{a+1}\right) .
\end{aligned}
$$

(Note that if $j(k-r) \equiv t(\bmod p)$ then $(j(k-r))^{p^{a}} \equiv t^{p^{a}}\left(\bmod p^{a+1}\right)$.)

Choose a primitive root $g$ modulo $p$. Since

$$
\left(g^{p^{a} m}-1\right) \sum_{j=1}^{p-1} j^{p^{a} m}=\sum_{j=1}^{p-1}(g j)^{p^{a} m}-\sum_{t=1}^{p-1} t^{p^{a} m} \equiv 0\left(\bmod p^{a+1}\right),
$$

if $p-1 \nmid m$ then $g^{p^{a} m}-1 \not \equiv 0(\bmod p)$ and so $\sum_{j=1}^{p-1} j^{p^{a} m} \equiv 0\left(\bmod p^{a+1}\right)$. Thus, when $k \equiv r(\bmod p)$ we have

$$
S_{k-r}=\sum_{j=1}^{p-1} j^{p^{a} m} \equiv(p-1) \llbracket p-1 \mid m \rrbracket\left(\bmod p^{a+1}\right) .
$$

Recall that $p / \pi^{p-1} \equiv-1(\bmod \pi)$. In view of the above,

$$
\begin{aligned}
\pi^{-p^{a} m} p C_{p}(n, r)-\sum_{k=0}^{n_{0}}\left(\begin{array}{c}
n_{0} \\
k
\end{array}\right)(-1)^{k}(k-r)^{p^{a} m^{*}} G\left(p^{a} m\right) \\
\equiv \sum_{\substack{k=0 \\
p \mid k-r}}^{n_{0}}\left(\begin{array}{c}
n_{0} \\
k
\end{array}\right)(-1)^{k}\left(\llbracket p-1 \mid m \rrbracket(p-1)-(k-r)^{p^{a} m^{*}} G\left(p^{a} m\right)\right) \\
\equiv C_{p}\left(n_{0}, r\right) \llbracket p-1 \mid m \rrbracket p\left(\bmod p^{a} \pi^{\min \left\{n_{0}+1, p-1\right\}}\right),
\end{aligned}
$$

where we have noted that if $p-1 \mid m$ (i.e., $m^{*}=0$ ) then

$$
p-1-G\left(p^{a} m\right) \equiv p-\sum_{t=0}^{p-1} \zeta_{p}^{t}=p-\frac{1-\zeta_{p}^{p}}{1-\zeta_{p}}=p\left(\bmod p^{a+1}\right) .
$$

Therefore the desired congruence follows.

Proof of Theorem 1.1. In the case $n=0,(1.2)$ holds since $n_{1}=n_{0}=0$ and $F_{p}(n, r)=-p C_{p}(0, r)+1$. Below we assume $n>0$.

Let $\zeta_{p}$ be a primitive $p$ th root of unity in $\mathbb{C}$, and set $\pi=1-\zeta_{p}$. By Lemma 2.2 in the case $a=1$,

$$
\begin{aligned}
& \pi^{-p\lfloor n / p\rfloor} C_{p}(n, r)-\llbracket n_{1}=0 \rrbracket C_{p}\left(n_{0}, r\right) \\
& \equiv \frac{G(p\lfloor n / p\rfloor)}{p} \sum_{k=0}^{n_{0}}\left(\begin{array}{c}
n_{0} \\
k
\end{array}\right)(-1)^{k}(k-r)^{p n_{1}}\left(\bmod \pi^{\min \left\{n_{0}+1, p-1\right\}}\right) .
\end{aligned}
$$


In view of Lemma 2.1,

$$
G\left(p\left\lfloor\frac{n}{p}\right\rfloor\right) \equiv G\left(\left\lfloor\frac{n}{p}\right\rfloor\right) \equiv(-1)^{n_{1}-1} \sum_{m=n_{1}}^{p-2} s\left(m, n_{1}\right) \frac{\pi^{m}}{m !}(\bmod p) .
$$

If $n_{0}>n_{1}$, then

$$
\sum_{k=0}^{n_{0}}\left(\begin{array}{c}
n_{0} \\
k
\end{array}\right)(-1)^{k}(k-r)^{p n_{1}} \equiv \sum_{k=0}^{n_{0}}\left(\begin{array}{c}
n_{0} \\
k
\end{array}\right)(-1)^{k}(k-r)^{n_{1}}=0(\bmod p),
$$

where we have applied Fermat's little theorem and Euler's identity (mentioned in Section 1). Therefore

$$
\begin{aligned}
& \pi^{-p\lfloor n / p\rfloor} C_{p}(n, r)-\llbracket n_{1}=0 \rrbracket C_{p}\left(n_{0}, r\right) \\
& \equiv \frac{(-1)^{n_{1}-1}}{p} \sum_{m=n_{1}}^{p-2} s\left(m, n_{1}\right) \frac{\pi^{m}}{m !} \sum_{k=0}^{n_{0}}\left(\begin{array}{c}
n_{0} \\
k
\end{array}\right)(-1)^{k}(k-r)^{p n_{1}} \\
& \quad\left(\bmod \pi^{\llbracket n_{0}>n_{1} \rrbracket \min \left\{n_{0}+1, p-1\right\}}\right) .
\end{aligned}
$$

Recall that $-p / \pi^{p-1} \equiv 1(\bmod \pi)$. Since $s\left(n_{1}, n_{1}\right)=1$ and

$$
\frac{p^{\llbracket n_{0} \leq n_{1} \rrbracket}}{\pi^{n_{1}}} \pi^{\llbracket n_{0}>n_{1} \rrbracket \min \left\{n_{0}+1, p-1\right\}} \equiv 0(\bmod \pi),
$$

by the above we have

$$
\begin{aligned}
& \frac{p^{\llbracket n_{0} \leq n_{1} \rrbracket} C_{p}(n, r)}{\pi^{p\lfloor n / p\rfloor+n_{1}}}-p^{\llbracket n_{0}=0 \rrbracket} \llbracket n_{1}=0 \rrbracket C_{p}\left(n_{0}, r\right) \\
& \equiv \frac{(-1)^{n_{1}-1} / n_{1} !}{p^{\llbracket n_{0}>n_{1} \rrbracket}} \sum_{k=0}^{n_{0}}\left(\begin{array}{c}
n_{0} \\
k
\end{array}\right)(-1)^{k}(k-r)^{p n_{1}}(\bmod \pi) .
\end{aligned}
$$

Note that

$$
\left\lfloor\frac{n-1}{p-1}\right\rfloor=\left\lfloor\frac{p\lfloor n / p\rfloor+n_{0}-1}{p-1}\right\rfloor=\frac{p\lfloor n / p\rfloor+n_{1}}{p-1}-\llbracket n_{0} \leq n_{1} \rrbracket
$$

and hence

$$
\begin{aligned}
\frac{(-p)^{\llbracket n_{0} \leq n_{1} \rrbracket} C_{p}(n, r)}{\pi^{p\lfloor n / p\rfloor+n_{1}}} & =\frac{C_{p}(n, r)}{(-p)^{\lfloor(n-1) /(p-1)\rfloor}}\left(\frac{-p}{\pi^{p-1}}\right)^{\left(p\lfloor n / p\rfloor+n_{1}\right) /(p-1)} \\
& \equiv F_{p}(n, r)(\bmod \pi) .
\end{aligned}
$$

In view of the above,

$$
\begin{aligned}
(-1)^{\left[n_{0} \leq n_{1}\right]} F_{p}(n, r)-\llbracket n_{0}>n_{1}=0 \rrbracket C_{p}\left(n_{0}, r\right) & \\
\equiv & \frac{(-1)^{n_{1}-1} / n_{1} !}{p^{\llbracket n_{0}>n_{1} \rrbracket}} \sum_{k=0}^{n_{0}}\left(\begin{array}{c}
n_{0} \\
k
\end{array}\right)(-1)^{k}(k-r)^{p n_{1}}(\bmod \pi) .
\end{aligned}
$$


As the rational $p$-adic integer

$$
\begin{aligned}
D= & F_{p}(n, r)-\llbracket n_{0}>n_{1}=0 \rrbracket C_{p}\left(n_{0}, r\right) \\
& -\frac{(-1)^{n_{1}}}{(-p)^{\llbracket n_{0}>n_{1} \rrbracket} \cdot n_{1} !} \sum_{k=0}^{n_{0}}\left(\begin{array}{c}
n_{0} \\
k
\end{array}\right)(-1)^{k}(k-r)^{p n_{1}}
\end{aligned}
$$

is divisible by $\pi$, we have $D^{p-1} \equiv 0(\bmod p)$ and hence $D \equiv 0(\bmod p)$. Thus

$$
\begin{aligned}
F_{p}(n, r)- & \llbracket n_{0}>n_{1}=0 \rrbracket C_{p}\left(n_{0}, r\right) \\
& \equiv \frac{(-1)^{n_{1}}}{(-p)^{\llbracket n_{0}>n_{1} \rrbracket} \cdot n_{1} !} \sum_{k=0}^{n_{0}}\left(\begin{array}{c}
n_{0} \\
k
\end{array}\right)(-1)^{k}(k-r)^{p n_{1}}(\bmod p) .
\end{aligned}
$$

In the case $n_{0} \leq n_{1},(2.2)$ reduces to (1.2). When $n_{0}>n_{1}=0$, (2.2) yields $(1.3)$ since $C_{p}\left(n_{0}, r\right)=(-1)^{\{r\}_{p}}\left(\begin{array}{c}n_{0} \\ \{r\}_{p}\end{array}\right)$ and $\sum_{k=0}^{n_{0}}\left(\begin{array}{c}n_{0} \\ k\end{array}\right)(-1)^{k}=$ $(1-1)^{n_{0}}=0$.

Now assume that $n_{0}>n_{1}>0$. As $\sum_{k=0}^{n_{0}}\left(\begin{array}{c}n_{0} \\ k\end{array}\right)(k-r)^{n_{1}}=0$ by Euler's identity, (2.2) implies that

$$
F_{p}(n, r) \equiv \frac{(-1)^{n_{1}-1}}{n_{1} !} \sum_{k=0}^{n_{0}}\left(\begin{array}{c}
n_{0} \\
k
\end{array}\right)(-1)^{k} \frac{(k-r)^{p n_{1}}-(k-r)^{n_{1}}}{p}(\bmod p) .
$$

If $n_{1}=1$, then

$$
\frac{(k-r)^{p n_{1}}-(k-r)^{n_{1}}}{p}=(k-r)^{n_{1}} n_{1} q_{p}(k-r) ;
$$

if $n_{1} \geq 2$ and $k \equiv r(\bmod p)$, then

$$
\frac{(k-r)^{p n_{1}}-(k-r)^{n_{1}}}{p} \equiv 0 \equiv(k-r)^{n_{1}} n_{1} q_{p}(k-r)(\bmod p) ;
$$

if $a=k-r \not \equiv 0(\bmod p)$, then

$$
\frac{(k-r)^{p n_{1}}-(k-r)^{n_{1}}}{p}=a^{n_{1}} \frac{\left(1+p \cdot q_{p}(a)\right)^{n_{1}}-1}{p} \equiv a^{n_{1}} n_{1} q_{p}(a)(\bmod p) .
$$

Therefore (1.4) follows.

3. Proof of Theorem 1.2. The following lemma is a refinement of an induction technique used by Sun [S06].

Lemma 3.1. Let $p$ be a prime, and let $n \in \mathbb{N}$ with $n \geq p$. Then

$$
F_{p}(n, r) \equiv-\sum_{j=1}^{p-1} \frac{1}{j} \sum_{i=0}^{j-1} F_{p}(n-p+1, r-i)(\bmod p) .
$$


Proof. Set $n^{\prime}=n-(p-1)>0$. By the Chu-Vandermonde convolution identity (cf. [GKP, (5.27)]),

$$
\begin{aligned}
F_{p}(n, r) & =(-p)^{-\lfloor(n-1) /(p-1)\rfloor} \sum_{\substack{0 \leq k \leq n \\
k \equiv r(\bmod p)}} \sum_{j=0}^{k}\left(\begin{array}{c}
p-1 \\
j
\end{array}\right)\left(\begin{array}{c}
n^{\prime} \\
k-j
\end{array}\right)(-1)^{k} \\
& =-\frac{1}{p} \sum_{j=0}^{p-1}\left(\begin{array}{c}
p-1 \\
j
\end{array}\right)(-p)^{-\left\lfloor\left(n^{\prime}-1\right) /(p-1)\right\rfloor} \sum_{\substack{j \leq k \leq n \\
p \mid k-r}}\left(\begin{array}{c}
n^{\prime} \\
k-j
\end{array}\right)(-1)^{k} \\
& =-\frac{1}{p} \sum_{j=0}^{p-1}\left(\begin{array}{c}
p-1 \\
j
\end{array}\right)(-1)^{j} F_{p}\left(n^{\prime}, r-j\right) .
\end{aligned}
$$

For any $j=0, \ldots, p-1$, clearly

$$
\begin{aligned}
\left(\begin{array}{c}
p-1 \\
j
\end{array}\right)(-1)^{j} & =\prod_{0<i \leq j}\left(1-\frac{p}{i}\right) \\
& \equiv 1-\sum_{0<i \leq j} \frac{p}{i} \equiv(-1)^{p-1}+p \sum_{j<k<p} \frac{1}{k}\left(\bmod p^{2}\right) .
\end{aligned}
$$

(Note that $\left.2 \sum_{k=1}^{p-1} 1 / k=\sum_{k=1}^{p-1}(1 / k+1 /(p-k)) \equiv 0(\bmod p).\right)$ Also,

$$
\sum_{j=0}^{p-1} F_{p}\left(n^{\prime}, r-j\right)=(-p)^{-\left\lfloor\left(n^{\prime}-1\right) /(p-1)\right\rfloor} \sum_{k=0}^{n^{\prime}}\left(\begin{array}{c}
n^{\prime} \\
k
\end{array}\right)(-1)^{k}=0 .
$$

Therefore

$$
F_{p}(n, r) \equiv-\sum_{j=0}^{p-1} \sum_{j<k<p} \frac{F_{p}\left(n^{\prime}, r-j\right)}{k}=-\sum_{k=1}^{p-1} \frac{1}{k} \sum_{j=0}^{k-1} F_{p}\left(n^{\prime}, r-j\right)(\bmod p) .
$$

This proves (3.1).

Proof of Theorem 1.2. (i) Suppose $m \geq 0$. Then

$$
\begin{aligned}
\sum_{k=0}^{n^{*}} \bar{S}(m & \left.+n^{*}-k, m\right) \frac{(-r)^{k}}{k !} \\
& =\left[x^{m+n^{*}}\right] \sum_{l=m}^{\infty} \bar{S}(l, m) x^{l} \sum_{k=0}^{\infty} \frac{(-r x)^{k}}{k !}=\left[x^{m+n^{*}}\right]\left(e^{x}-1\right)^{m} e^{-r x} \\
& =\left[x^{n^{*}}\right]\left(\frac{e^{x}-1}{x}\right)^{m} e^{-r x}=\left[x^{m+n^{*}}\right] \sum_{k=0}^{m}\left(\begin{array}{c}
m \\
k
\end{array}\right)(-1)^{m-k} e^{(k-r) x} \\
& =\sum_{k=0}^{m}\left(\begin{array}{c}
m \\
k
\end{array}\right)(-1)^{m-k} \frac{(k-r)^{m+n^{*}}}{\left(m+n^{*}\right) !} .
\end{aligned}
$$


By the identity (2.4) of Sun [S03], for any $l=0,1, \ldots$ we have

$$
\begin{aligned}
\sum_{k=0}^{m}\left(\begin{array}{c}
m \\
k
\end{array}\right)(-1)^{m-k}(k+l)^{m+n^{*}} & =\sum_{j=0}^{l}\left(\begin{array}{l}
l \\
j
\end{array}\right)(m+j) ! S\left(m+n^{*}, m+j\right) \\
& =\sum_{j=0}^{n^{*}}\left(\begin{array}{l}
l \\
j
\end{array}\right)(m+j) ! S\left(m+n^{*}, m+j\right) .
\end{aligned}
$$

Thus

$$
\sum_{k=0}^{m}\left(\begin{array}{c}
m \\
k
\end{array}\right)(-1)^{m-k}(k+x)^{m+n^{*}}=\sum_{j=0}^{n^{*}}\left(\begin{array}{l}
x \\
j
\end{array}\right)(m+j) ! S\left(m+n^{*}, m+j\right)
$$

and hence

$$
\sum_{k=0}^{m}\left(\begin{array}{c}
m \\
k
\end{array}\right)(-1)^{m-k} \frac{(k-r)^{m+n^{*}}}{\left(m+n^{*}\right) !}=\sum_{j=0}^{n^{*}}\left(\begin{array}{c}
-r \\
j
\end{array}\right) \bar{S}\left(m+n^{*}, m+j\right) .
$$

If $m \leq 0$, then

$$
\frac{B_{n^{*}}^{(-m)}(-r)}{n^{*} !}=\left[x^{n^{*}}\right]\left(\frac{x}{e^{x}-1}\right)^{-m} e^{-r x}=\left[x^{n^{*}}\right]\left(\frac{e^{x}-1}{x}\right)^{m} e^{-r x} .
$$

Note also that

$$
\frac{1}{n^{*} !}=\frac{\prod_{j=1}^{p-1-n^{*}}(p-j)}{(p-1) !} \equiv(-1)^{n^{*}+1}\left(p-1-n^{*}\right) !(\bmod p)
$$

by Wilson's theorem.

In view of the above, whether $m \geq 0$ or $m \leq 0$, we only need to show that

$$
(-1)^{n} F_{p}(n, r) \equiv\left[x^{n^{*}}\right]\left(\frac{e^{x}-1}{x}\right)^{m} e^{-r x}(\bmod p) .
$$

(ii) All those formal power series $f(x)=\sum_{k=0}^{\infty} a_{k} x^{k}$ with $a_{k} \in \mathbb{Q}$ and $a_{0}, \ldots, a_{n^{*}} \in \mathbb{Z}_{p}$ form a ring $R_{n^{*}}$ under the usual addition and multiplication. In particular, this ring contains

$$
e^{-r x}=\sum_{k=0}^{\infty}(-r)^{k} \frac{x^{k}}{k !}, \quad \frac{e^{x}-1}{x}=\sum_{k=0}^{\infty} \frac{x^{k}}{(k+1) !}, \quad \frac{x}{e^{x}-1}=\sum_{k=0}^{\infty} B_{k} \frac{x^{k}}{k !} .
$$

(Recall that $n^{*}<p-1$ and $B_{0}, \ldots, B_{n^{*}} \in \mathbb{Z}_{p}$.) If $f(x)=\sum_{k=0}^{\infty} a_{k} x^{k}$ and 
$g(x)=\sum_{k=0}^{\infty} b_{k} x^{k}$ belong to $R_{n^{*}}$, then

$$
\begin{aligned}
{\left[x^{n^{*}}\right] f(x) g(x)^{p} } & =\left[x^{n^{*}}\right] \sum_{j=0}^{n^{*}} a_{j} x^{j}\left(\sum_{k=0}^{n^{*}} b_{k} x^{k}\right)^{p} \\
& \equiv\left[x^{n^{*}}\right] \sum_{j=0}^{n^{*}} a_{j} x^{j} \sum_{k=0}^{n^{*}} b_{k}^{p} x^{p k}=a_{n^{*}} b_{0}^{p} \\
& \equiv\left[x^{n^{*}}\right] f(x)\left[x^{0}\right] g(x)(\bmod p) .
\end{aligned}
$$

Consequently, for any $a \in \mathbb{Z}$ we have

$$
\left[x^{n^{*}}\right]\left(\frac{e^{x}-1}{x}\right)^{m} e^{a x} \equiv\left[x^{n^{*}}\right]\left(\frac{e^{x}-1}{x}\right)^{n} e^{a x}(\bmod p)
$$

since $m \equiv n(\bmod p)$. From this and part $(\mathrm{i})$, it suffices to use induction on $n$ to show that

$$
(-1)^{n} F_{p}(n, r) \equiv\left[x^{n^{*}}\right]\left(\frac{e^{x}-1}{x}\right)^{n} e^{-r x}(\bmod p) .
$$

(iii) Obviously

$$
(-1)^{0} F_{p}(0, r)=-p C_{p}(0, r)+1 \equiv 1=\left[x^{0}\right]\left(\frac{e^{x}-1}{x}\right)^{0} e^{-r x}(\bmod p) .
$$

So (3.2) holds for $n=0$.

Suppose that $0<n \leq p-1$. Then $n^{*}=p-1-n$ and

$$
\begin{aligned}
& {\left[x^{n^{*}}\right]\left(\frac{e^{x}-1}{x}\right)^{n} e^{-r x}=\left[x^{p-1}\right]\left(e^{x}-1\right)^{n} e^{-r x}} \\
& \quad=\sum_{k=0}^{n}\left(\begin{array}{l}
n \\
k
\end{array}\right)(-1)^{n-k}\left[x^{p-1}\right] e^{(k-r) x}=\sum_{k=0}^{n}\left(\begin{array}{l}
n \\
k
\end{array}\right)(-1)^{n-k} \frac{(k-r)^{p-1}}{(p-1) !} \\
& \quad \equiv(-1)^{n-1} \sum_{k \neq r(\bmod p)}\left(\begin{array}{l}
n \\
k
\end{array}\right)(-1)^{k}(\bmod p) .
\end{aligned}
$$

(To get the last congruence we have applied Wilson's theorem and Fermat's little theorem.) Since

$$
-\sum_{k \neq \equiv(\bmod p)}\left(\begin{array}{l}
n \\
k
\end{array}\right)(-1)^{k}=\sum_{k \equiv r(\bmod p)}\left(\begin{array}{l}
n \\
k
\end{array}\right)(-1)^{k}=F_{p}(n, r),
$$

the desired (3.2) follows. 
Now fix $n \geq p$ and assume that (3.2) holds for smaller values of $n$. Clearly $n^{\prime}=n-(p-1)>0$ and $\left\{-n^{\prime}\right\}_{p-1}=n^{*}$. In light of Lemma 3.1,

$$
F_{p}(n, r) \equiv-\sum_{j=1}^{p-1} \frac{1}{j} \sum_{k=0}^{j-1} F_{p}\left(n^{\prime}, r-k\right)(\bmod p) .
$$

By the induction hypothesis and part (ii),

$$
\begin{aligned}
(-1)^{n^{\prime}} F_{p}\left(n^{\prime}, r-k\right) & \equiv\left[x^{n^{*}}\right]\left(\frac{e^{x}-1}{x}\right)^{n^{\prime}} e^{-(r-k) x} \\
& \equiv\left[x^{n^{*}}\right]\left(\frac{e^{x}-1}{x}\right)^{n+1} e^{(k-r) x}(\bmod p) .
\end{aligned}
$$

Thus $(-1)^{n-1} F_{p}(n, r)$ is congruent to

$$
\begin{aligned}
\sum_{j=1}^{p-1} \frac{1}{j} \sum_{k=0}^{j-1}\left(\left[x^{n^{*}}\right]\left(\frac{e^{x}-1}{x}\right)^{n+1} e^{(k-r) x}\right) & \\
= & {\left[x^{n^{*}}\right]\left(\frac{e^{x}-1}{x}\right)^{n+1} e^{-r x} \sum_{j=1}^{p-1}\left(\frac{1}{j} \cdot \frac{e^{j x}-1}{e^{x}-1}\right) } \\
& =\left[x^{n^{*}}\right]\left(\frac{e^{x}-1}{x}\right)^{n} e^{-r x} \sum_{j=1}^{p-1} \frac{e^{j x}-1}{j x}
\end{aligned}
$$

modulo $p$. This yields

$$
\begin{aligned}
(-1)^{n} F_{p}(n, r) & \equiv-\left[x^{n^{*}}\right]\left(\frac{e^{x}-1}{x}\right)^{n} e^{-r x} \sum_{j=1}^{p-1} \sum_{k=1}^{p-1} \frac{(j x)^{k-1}}{k !} \\
& \equiv\left[x^{n^{*}}\right]\left(\frac{e^{x}-1}{x}\right)^{n} e^{-r x}(\bmod p)
\end{aligned}
$$

since $n^{*}<p-1$ and $\sum_{j=1}^{p-1} j^{k-1} \equiv-\llbracket p-1 \mid k-1 \rrbracket(\bmod p)$.

In view of the above, we have completed the proof.

4. Proof of Theorem 1.3. Let $\zeta_{p}$ be a primitive $p$ th root of unity in $\mathbb{C}$, and set $\pi=1-\zeta_{p}$. For any $k=0, \ldots, n$, we have

$$
\begin{aligned}
p C_{p}\left(k p^{a}(p-1)+l, r\right) & =\sum_{j=0}^{p-1} \zeta_{p}^{-j r}\left(1-\zeta_{p}^{j}\right)^{k p^{a}(p-1)+l} \\
& =\sum_{j=1}^{p-1} \zeta_{p}^{-j r}\left(1-\zeta_{p}^{j}\right)^{k p^{a}(p-1)+l}+\llbracket k=l=0 \rrbracket
\end{aligned}
$$


and thus

$$
\begin{aligned}
& F_{p}\left(k p^{a}(p-1)+l, r\right) \\
& \quad=(-p)^{-\left\lfloor\left(k p^{a}(p-1)+l-1\right) /(p-1)\right\rfloor} C_{p}\left(k p^{a}(p-1)+l, r\right)+\llbracket k=l=0 \rrbracket \\
& \quad=-(-p)^{-k p^{a}-\lfloor(l-1) /(p-1)\rfloor-1} \sum_{j=1}^{p-1} \zeta_{p}^{-j r}\left(1-\zeta_{p}^{j}\right)^{k p^{a}(p-1)+l} .
\end{aligned}
$$

Therefore, for $S_{n}=\sum_{k=0}^{n}\left(\begin{array}{l}n \\ k\end{array}\right)(-1)^{k} F_{p}\left(k p^{a}(p-1)+l, r\right)$ we have

$$
S_{n}=-\sum_{j=1}^{p-1} \zeta_{p}^{-j r}\left(1-\zeta_{p}^{j}\right)^{l}(-p)^{-\lfloor(l-1) /(p-1)\rfloor-1} c_{n, j},
$$

where

$$
\begin{aligned}
c_{n, j} & =\sum_{k=0}^{n}\left(\begin{array}{l}
n \\
k
\end{array}\right)(-1)^{k}(-p)^{-k p^{a}}\left(1-\zeta_{p}^{j}\right)^{k p^{a}(p-1)} \\
& =\left(1-(-p)^{-p^{a}}\left(1-\zeta_{p}^{j}\right)^{p^{a}(p-1)}\right)^{n} .
\end{aligned}
$$

Let $j \in\{1, \ldots, p-1\}$. Clearly

$$
\left(\frac{1-\zeta_{p}^{j}}{\pi}\right)^{p-1}=\left(\frac{1-(1-\pi)^{j}}{\pi}\right)^{p-1} \equiv j^{p-1} \equiv 1(\bmod \pi)
$$

and hence

$$
b_{j}:=\frac{\left(1-\zeta_{p}^{j}\right)^{p-1}}{-p}=\left(\frac{1-\zeta_{p}^{j}}{\pi}\right)^{p-1} \frac{\pi^{p-1}}{-p} \equiv 1(\bmod \pi) .
$$

(Recall the congruence $p / \pi^{p-1} \equiv-1(\bmod \pi)$.) It follows that $b_{j}^{p^{a}} \equiv 1$ $\left(\bmod p^{a} \pi\right)$ and

$$
c_{n, j}=\left(1-b_{j}^{p^{a}}\right)^{n} \equiv 0\left(\bmod p^{a n} \pi^{n}\right) .
$$

Since $\left(1-\zeta_{p}^{j}\right)^{l} \equiv 0\left(\bmod \pi^{l}\right)$ and $\operatorname{ord}_{p}(\pi)=1 /(p-1)$, in view of $(4.1)$ and (4.2) we have $\operatorname{ord}_{p}\left(S_{n}\right) \geq \frac{l+n}{p-1}+a n-\left\lfloor\frac{l-1}{p-1}\right\rfloor-1=a n+\frac{l+n}{p-1}-\frac{l+l^{*}}{p-1}=a n+\frac{n-l^{*}}{p-1}$ and hence $\operatorname{ord}_{p}\left(S_{n}\right) \geq a n+\left\lceil\left(n-l^{*}\right) /(p-1)\right\rceil$. This proves (1.20).

\section{On generalized Fleck quotients}

Lemma 5.1. Let $d, q \in \mathbb{Z}^{+}, n \in \mathbb{N}$ and $r \in \mathbb{Z}$. Let $\zeta_{d q}$ be a primitive dqth root of unity in $\mathbb{C}$. Then

$$
C_{d q}(n, r)=\frac{1}{d} \sum_{k=0}^{n}\left(\begin{array}{l}
n \\
k
\end{array}\right) C_{q}(k, r) \sum_{j=0}^{d-1} \zeta_{d q}^{j(k-r)}\left(1-\zeta_{d q}^{j}\right)^{n-k} .
$$


Proof. Note that $\zeta=\zeta_{d q}^{d}$ is a primitive $q$ th root of unity. Thus

$$
\begin{aligned}
q \sum_{k=0}^{n}\left(\begin{array}{l}
n \\
k
\end{array}\right) C_{q}(k, r) \sum_{j=0}^{d-1} \zeta_{d q}^{j(k-r)}\left(1-\zeta_{d q}^{j}\right)^{n-k} \\
=\sum_{k=0}^{n}\left(\begin{array}{l}
n \\
k
\end{array}\right) \sum_{s=0}^{q-1} \zeta^{-s r}\left(1-\zeta^{s}\right)^{k} \sum_{j=0}^{d-1} \zeta_{d q}^{j(k-r)}\left(1-\zeta_{d q}^{j}\right)^{n-k} \\
=\sum_{s=0}^{q-1} \sum_{j=0}^{d-1} \zeta_{d q}^{-(d s+j) r} \sum_{k=0}^{n}\left(\begin{array}{l}
n \\
k
\end{array}\right)\left(\zeta_{d q}^{j}\left(1-\zeta_{d q}^{d s}\right)\right)^{k}\left(1-\zeta_{d q}^{j}\right)^{n-k} \\
=\sum_{s=0}^{q-1} \sum_{j=0}^{d-1} \zeta_{d q}^{-(d s+j) r}\left(1-\zeta_{d q}^{d s+j}\right)^{n}=\sum_{t=0}^{d q-1} \zeta_{d q}^{-t r}\left(1-\zeta_{d q}^{t}\right)^{n}=d q C_{d q}(n, r)
\end{aligned}
$$

So we have (5.1).

With the help of Lemma 5.1 we can prove the following result via roots of unity.

Theorem 5.1 (Weisman, 1977). Let $p$ be a prime, and let $a \in \mathbb{Z}^{+}, n \in \mathbb{N}$ and $r \in \mathbb{Z}$. Then $F_{p^{a}}(n, r) \in \mathbb{Z}$.

Proof. We use induction on $a$.

The case $a=1$ reduces to Fleck's result. A proof of Fleck's result via roots of unity was given by A. Granville [Gr].

Now let $a \geq 2$ and assume that $F_{p^{a-1}}\left(n^{\prime}, r^{\prime}\right) \in \mathbb{Z}$ for all $n^{\prime} \in \mathbb{N}$ and $r^{\prime} \in \mathbb{Z}$. If $n<p^{a}$, then $\left\lfloor\left(n-p^{a-1}\right) / \varphi\left(p^{a}\right)\right\rfloor \leq 0$ and hence $F_{p^{a}}(n, r) \in \mathbb{Z}$. Below we suppose $n \geq p^{a}$ and let $\zeta_{p^{a}}$ be a primitive $p^{a}$ th root of unity in $\mathbb{C}$.

By Lemma 5.1,

$$
C_{p^{a}}(n, r)=\frac{1}{p} \sum_{k=0}^{n}\left(\begin{array}{l}
n \\
k
\end{array}\right) C_{p^{a-1}}(k, r) \sum_{j=0}^{p-1} \zeta_{p^{a}}^{j(k-r)}\left(1-\zeta_{p^{a}}^{j}\right)^{n-k} .
$$

Observe that

$$
\prod_{\substack{j=1 \\ p \nmid j}}^{p^{a}-1}\left(1-\zeta_{p^{a}}^{j}\right)=\prod_{\substack{\gamma^{p^{a}}=1 \\ \gamma^{p^{a}} \neq 1}}(1-\gamma)=\lim _{x \rightarrow 1} \frac{x^{p^{a}}-1}{x^{p^{a-1}}-1}=\frac{p^{a}}{p^{a-1}}=p .
$$

If $p \nmid j$, then $\left(1-\zeta_{p^{a}}^{j}\right) /\left(1-\zeta_{p^{a}}\right)$ is a unit in the ring $\mathbb{Z}\left[\zeta_{p^{a}}\right]$ and thus

$$
\operatorname{ord}_{p}\left(1-\zeta_{p^{a}}^{j}\right)=\operatorname{ord}_{p}\left(1-\zeta_{p^{a}}\right)=\frac{1}{\varphi\left(p^{a}\right)} .
$$


From this and the induction hypothesis, for any $k=0, \ldots, n$ we have

$$
\begin{aligned}
& \operatorname{ord}_{p}\left(C_{p^{a-1}}(k, r) \sum_{j=0}^{p-1} \zeta_{p^{a}}^{j(k-r)}\left(1-\zeta_{p^{a}}^{j}\right)^{n-k}\right) \\
& \left.\geq \max \left\{0, \mid \frac{k-p^{a-2}}{\varphi\left(p^{a-1}\right)}\right\rfloor\right\}+\frac{n-k}{\varphi\left(p^{a}\right)} \\
& \quad=\max \left\{0, \frac{p k-p^{a-1}}{\varphi\left(p^{a}\right)}-\left\{\frac{k-p^{a-2}}{\varphi\left(p^{a-1}\right)}\right\}\right\}+\frac{n-k}{\varphi\left(p^{a}\right)} \\
& \quad=\max \left\{\frac{n-k}{\varphi\left(p^{a}\right)}, \frac{n-p^{a-1}}{\varphi\left(p^{a}\right)}+\frac{k}{p^{a-1}}-\left\{\frac{k-p^{a-2}}{\varphi\left(p^{a-1}\right)}\right\}\right\}>\frac{n-p^{a-1}}{\varphi\left(p^{a}\right)} .
\end{aligned}
$$

(Note that if $k \geq p^{a-1}$ then $k / p^{a-1} \geq 1>\left\{\left(k-p^{a-2}\right) / \varphi\left(p^{a-1}\right)\right\}$.) Therefore, from (5.2) we get

$$
\operatorname{ord}_{p}\left(C_{p^{a}}(n, r)\right)>\frac{n-p^{a-1}}{\varphi\left(p^{a}\right)}-1 \geq\left\lfloor\frac{n-p^{a-1}}{\varphi\left(p^{a}\right)}\right\rfloor-1 .
$$

So $F_{p^{a}}(n, r)=(-p)^{-\left\lfloor\left(n-p^{a-1}\right) / \varphi\left(p^{a}\right)\right\rfloor} C_{p^{a}}(n, r) \in \mathbb{Z}$ as desired.

Proof of Theorem 1.4. (i) Write $n+d=p^{a-1}-1+m \varphi\left(p^{a}\right)$ with $m \in \mathbb{N}$. Then, for any $k=0, \ldots, d$ we have

$$
\left\lfloor\frac{n+k-p^{a-1}}{\varphi\left(p^{a}\right)}\right\rfloor=\left\lfloor m-\frac{d-k+1}{\varphi\left(p^{a}\right)}\right\rfloor=m-1 .
$$

Below we use induction on $d$ to show the desired congruence (1.21).

In the case $d=0$ (i.e., $\left.n-p^{a-1} \equiv-1\left(\bmod \varphi\left(p^{a}\right)\right)\right)$, we have $F_{p^{a}}(n, r) \equiv$ $F_{p^{a}}(n, 0)(\bmod p)$ because

$$
F_{p^{a}}(n, i)-F_{p^{a}}(n, i-1)=(-p)^{-m+1} C_{p^{a}}(n+1, i)=-p F_{p^{a}}(n+1, i)
$$

for all $i \in \mathbb{Z}$. Furthermore, by a result of Weisman [We] (see also [SW, Theorem 1.5]), $F_{p^{a}}(n, r) \equiv 1(\bmod p)$ if $d=0$.

Now let $d>0$ and assume that the desired result holds for smaller values of $d$. Clearly, $(n+1)+(d-1)=p^{a-1}-1+m \varphi\left(p^{a}\right)$ and

$$
\left\lfloor\frac{n+1+k-p^{a-1}}{\varphi\left(p^{a}\right)}\right\rfloor=m-1 \quad \text { for } k=0, \ldots, d-1 .
$$

If $r \geq 0$ then

$$
C_{p^{a}}(n, r)-C_{p^{a}}(n, 0)=\sum_{0<i \leq r}\left(C_{p^{a}}(n, i)-C_{p^{a}}(n, i-1)\right)=\sum_{0<i \leq r} C_{p^{a}}(n+1, i) ;
$$


if $r<0$ then

$$
\begin{aligned}
C_{p^{a}}(n, r)-C_{p^{a}}(n, 0) & =\sum_{r<i \leq 0}\left(C_{p^{a}}(n, i-1)-C_{p^{a}}(n, i)\right) \\
& =-\sum_{r<i \leq 0} C_{p^{a}}(n+1, i) .
\end{aligned}
$$

Therefore

$$
F_{p^{a}}(n, r)-F_{p^{a}}(n, 0)= \begin{cases}\sum_{0<i \leq r} F_{p^{a}}(n+1, i) & \text { if } r \geq 0, \\ -\sum_{r<i \leq 0} F_{p^{a}}(n+1, i) & \text { if } r<0 .\end{cases}
$$

By the induction hypothesis, whenever $i \in \mathbb{Z}$ we have

$$
F_{p^{a}}(n+1, i) \equiv \sum_{k=0}^{d-1}\left(\begin{array}{c}
i+k-1 \\
k
\end{array}\right) F_{p^{a}}(n+1+k, 0)(\bmod p) .
$$

For any $k=0, \ldots, d-1$, if $r \geq 0$ then

$$
\sum_{0<i \leq r}\left(\begin{array}{c}
i+k-1 \\
k
\end{array}\right)=\sum_{j=0}^{r+k-1}\left(\begin{array}{l}
j \\
k
\end{array}\right)=\left(\begin{array}{l}
r+k \\
k+1
\end{array}\right)
$$

by an identity of S.-C. Chu (cf. [GKP, (5.10)]); if $r<0$ then

$$
\begin{aligned}
-\sum_{r<i \leq 0}\left(\begin{array}{c}
i+k-1 \\
k
\end{array}\right) & =(-1)^{k+1} \sum_{r<i \leq 0}\left(\begin{array}{c}
-i \\
k
\end{array}\right)=(-1)^{k+1} \sum_{j=0}^{-r-1}\left(\begin{array}{l}
j \\
k
\end{array}\right) \\
& =(-1)^{k+1}\left(\begin{array}{c}
-r \\
k+1
\end{array}\right)=\left(\begin{array}{c}
r+k \\
k+1
\end{array}\right) .
\end{aligned}
$$

Thus, by the above, $F_{p^{a}}(n, r)$ is congruent to

$$
F_{p^{a}}(n, 0)+\sum_{k=0}^{d-1}\left(\begin{array}{c}
r+k \\
k+1
\end{array}\right) F_{p^{a}}(n+1+k, 0)=\sum_{k=0}^{d}\left(\begin{array}{c}
r+k-1 \\
k
\end{array}\right) F_{p^{a}}(n+k, 0)
$$

modulo $p$. This concludes the induction proof of (1.21).

(ii) In the case $a=1$, the desired results in Theorem 1.4(ii) follow from Corollaries 1.3 and 1.7.

Now we let $a \geq 2$ and $r \in \mathbb{Z}$. Write $n=p^{a-2}\left(p n_{1}+n_{0}\right)+s$ and $r=$ $p^{a-2}\left(p r_{1}+r_{0}\right)+t$, where $s, t \in\left\{0, \ldots, p^{a-2}-1\right\}, n_{0}, r_{0} \in\{0, \ldots, p-1\}$ and $n_{1} \in \mathbb{N}$ and $r_{1} \in \mathbb{Z}$.

If $p^{a-1} \leq n<p^{a}$, then

$$
F_{p^{a}}(n, r)=C_{p^{a}}(n, r)=\left(\begin{array}{c}
n \\
\{r\}_{p^{a}}
\end{array}\right)(-1)^{\{r\}_{p^{a}}},
$$

and in particular $\operatorname{ord}_{p}\left(C_{p^{a}}(n, 0)\right)=0=\left\lfloor\left(n-p^{a-1}\right) / \varphi\left(p^{a}\right)\right\rfloor$. 
Below we assume that $n \geq 2 p^{a-1}$ (i.e., $n_{1} \geq 2$ ). By [SD, Theorem 1.7],

$$
F_{p^{a}}(n, r) \equiv(-1)^{t}\left(\begin{array}{l}
s \\
t
\end{array}\right) F_{p^{2}}\left(p n_{1}+n_{0}, p r_{1}+r_{0}\right)(\bmod p) .
$$

If $p \mid n_{1}$, or $p-1 \nmid n_{1}-1$, or $n_{0}=r_{0}=p-1$, then by [SW, Theorem 1.2] in the case $l=0$, we have

$$
F_{p^{2}}\left(p n_{1}+n_{0}, p r_{1}+r_{0}\right) \equiv(-1)^{r_{0}}\left(\begin{array}{c}
n_{0} \\
r_{0}
\end{array}\right) F_{p}\left(n_{1}, r_{1}\right)(\bmod p)
$$

and hence $F_{p^{a}}(n, r) \equiv b_{n, r} F_{p}\left(n_{1}, r_{1}\right)(\bmod p)$, where

$$
\begin{aligned}
b_{n, r} & :=(-1)^{\{r\}_{p^{a-1}}}\left(\begin{array}{c}
\{n\}_{p^{a-1}} \\
\{r\}_{p^{a-1}}
\end{array}\right)=(-1)^{p^{a-2} r_{0}+t}\left(\begin{array}{c}
p^{a-2} n_{0}+s \\
p^{a-2} r_{0}+t
\end{array}\right) \\
& \equiv(-1)^{t}\left(\begin{array}{l}
s \\
t
\end{array}\right)(-1)^{r_{0}}\left(\begin{array}{c}
n_{0} \\
r_{0}
\end{array}\right)(\bmod p) \quad \text { (by Lucas' theorem (cf. [HS])). }
\end{aligned}
$$

By Corollary 1.3, there is an $r_{1}^{\prime} \in \mathbb{Z}$ such that $F_{p}\left(n_{1}, r_{1}^{\prime}\right) \not \equiv 0(\bmod p)$. Thus, if $p \mid n_{1}$ or $p-1 \nmid n_{1}-1$, then

$$
F_{p^{a}}\left(n, p^{a-1} r_{1}^{\prime}\right) \equiv F_{p}\left(n_{1}, r_{1}^{\prime}\right) \not \equiv 0(\bmod p) .
$$

If $n_{0}=p-1$, then

$$
F_{p^{a}}\left(n, p^{a-2}\left(p r_{1}^{\prime}+p-1\right)\right) \equiv(-1)^{p-1}\left(\begin{array}{c}
p-1 \\
p-1
\end{array}\right) F_{p}\left(n_{1}, r_{1}^{\prime}\right) \not \equiv 0(\bmod p) .
$$

When $p \nmid n_{1}, p-1 \mid n_{1}-1$ and $n_{0}<r_{0}$, by applying the second part of [SW, Theorem 1.2] in the case $l=0$, we have

$$
F_{p^{2}}\left(p n_{1}+n_{0}, p r_{1}+r_{0}\right) \equiv \llbracket n_{1}>1 \rrbracket \frac{(-1)^{n_{0}} n_{1}}{r_{0}\left(\begin{array}{c}
r_{0}-1 \\
n_{0}
\end{array}\right)}=\frac{(-1)^{n_{0}} n_{1}}{r_{0}\left(\begin{array}{c}
r_{0}-1 \\
n_{0}
\end{array}\right)}(\bmod p)
$$

and hence

$$
F_{p^{a}}(n, r) \equiv(-1)^{n_{0}+t} \frac{n_{1}\left(\begin{array}{l}
s \\
t
\end{array}\right)}{r_{0}\left(\begin{array}{c}
r_{0}-1 \\
n_{0}
\end{array}\right)}(\bmod p) .
$$

In particular, if $p \nmid n_{1}, p-1 \mid n_{1}-1$ and $n_{0}<p-1$, then

$$
F_{p^{a}}\left(n, p^{a-2}\left(n_{0}+1\right)\right) \equiv \frac{(-1)^{n_{0}} n_{1}}{n_{0}+1} \not \equiv 0(\bmod p) .
$$

In view of the above, we already have (1.22).

To prove the congruence in (1.23), we also have to consider the case $p \nmid n_{1}, p-1 \mid n_{1}-1$ and $n_{0} \geq r_{0}$. By [SW, Lemmas 3.2 and 3.3], 


$$
\begin{aligned}
& p^{-\left\lfloor\left(p n_{1}+n_{0}-p\right) / \varphi\left(p^{2}\right)\right\rfloor} C_{p^{2}}\left(p n_{1}+n_{0}, p r_{1}+r_{0}\right) \\
& \quad-(-1)^{r_{0}}\left(\begin{array}{c}
n_{0} \\
r_{0}
\end{array}\right) p^{-\left\lfloor\left(n_{1}-1\right) /(p-1)\right\rfloor} C_{p}\left(n_{1}, r_{1}\right) \\
& \equiv(-1)^{n_{1}-1} p^{-\left\lfloor\left(n_{1}-1-1\right) /(p-1)\right\rfloor} C_{p}\left(n_{1}-1, r_{1}\right)(-1)^{n_{1}+r_{0}} n_{1}\left(\begin{array}{c}
n_{0} \\
r_{0}
\end{array}\right) \frac{\sigma_{n_{0}, r_{0}}\left(n_{1}\right)}{p} \\
& \equiv-(-1)^{r_{0}}\left(\begin{array}{c}
n_{0} \\
r_{0}
\end{array}\right) p^{-\left(n_{1}-1\right) /(p-1)+1} C_{p}\left(n_{1}-1, r_{1}\right) n_{1} \frac{\sigma_{n_{0}, r_{0}}\left(n_{1}\right)}{p}(\bmod p),
\end{aligned}
$$

where

$$
\sigma_{n_{0}, r_{0}}\left(n_{1}\right)=1+(-1)^{p} \frac{\prod_{1 \leq i \leq p, i \neq p-r_{0}}\left(p\left(n_{1}-1\right)+r_{0}+i\right)}{\prod_{1 \leq i \leq p, i \neq p-\left(n_{0}-r_{0}\right)}\left(n_{0}-r_{0}+i\right)} \equiv 0(\bmod p) .
$$

Therefore

$$
\begin{aligned}
& F_{p^{2}}\left(p n_{1}\right.\left.+n_{0}, p r_{1}+r_{0}\right)-(-1)^{r_{0}}\left(\begin{array}{c}
n_{0} \\
r_{0}
\end{array}\right) F_{p}\left(n_{1}, r_{1}\right) \\
& \equiv(-1)^{r_{0}}\left(\begin{array}{c}
n_{0} \\
r_{0}
\end{array}\right) F_{p}\left(n_{1}-1, r_{1}\right) n_{1} \frac{\sigma_{n_{0}, r_{0}}\left(n_{1}\right)}{p}(\bmod p)
\end{aligned}
$$

and hence

$$
F_{p^{a}}(n, r) \equiv b_{n, r}\left(F_{p}\left(n_{1}, r_{1}\right)+F_{p}\left(n_{1}-1, r_{1}\right) n_{1} \frac{\sigma_{n_{0}, r_{0}}\left(n_{1}\right)}{p}\right)(\bmod p) .
$$

Observe that $n+p^{a}(p-1)=p^{a-2}\left(p n_{1}^{\prime}+n_{0}\right)+s$ with $n_{1}^{\prime}=n_{1}+p(p-1)$. Clearly $F_{p}\left(n_{1}^{\prime}, r_{1}\right) \equiv F_{p}\left(n_{1}, r_{1}\right)(\bmod p)$ by Corollary 1.7 , and $\sigma_{n_{0}, r_{0}}\left(n_{1}^{\prime}\right) \equiv$ $\sigma_{n_{0}, r_{0}}\left(n_{1}\right)\left(\bmod p^{2}\right)$ if $n_{0} \geq r_{0}$. Thus, by the above, $F_{p^{a}}\left(n+p^{a}(p-1), r\right) \equiv$ $F_{p^{a}}(n, r)(\bmod p)$.

\section{References}

[BEW] B. C. Berndt, R. J. Evans and K. S. Williams, Gauss and Jacobi Sums, Wiley, New York, 1998.

[C] S. Chowla, On the class number of real quadratic fields, Proc. Nat. Acad. Sci. U.S.A. 47 (1961), 878.

[Co] P. Colmez, Une correspondance de Langlands locale p-adique pour les représentations semi-stables de dimension 2, preprint, 2004.

[DS] D. M. Davis and Z. W. Sun, A number-theoretic approach to homotopy exponents of SU(n), J. Pure Appl. Algebra 209 (2007), 57-69.

[D] L. E. Dickson, History of the Theory of Numbers, Vol. I, Chelsea, New York, 1999.

[G1] J. W. L. Glaisher, Congruences relating to the sums of products of the first n numbers and to other sums of products, Quart. J. Pure Appl. Math. 31 (1900), $1-35$.

[G2] - On the residues of the sums of products of the first $p-1$ numbers, and their powers, to modulus $p^{2}$ or $p^{3}$, ibid., 321-353. 
[GKP] R. L. Graham, D. E. Knuth and O. Patashnik, Concrete Mathematics, 2nd ed., Addison-Wesley, Reading, MA, 1994.

[Gr] A. Granville, Arithmetic properties of binomial coefficients. I. Binomial coefficients modulo prime powers, in: Organic Mathematics (Burnaby, BC, 1995), CMS Conf. Proc. 20, Amer. Math. Soc., Providence, RI, 1997, 253-276.

[HS] H. Hu and Z. W. Sun, An extension of Lucas' theorem, Proc. Amer. Math. Soc. 129 (2001), 3471-3478.

[IR] K. Ireland and M. Rosen, A Classical Introduction to Modern Number Theory, 2nd ed., Grad. Texts in Math. 84, Springer, New York, 1990.

[LW] J. H. van Lint and R. M. Wilson, A Course in Combinatorics, 2nd ed., Cambridge Univ. Press, Cambridge, 2001.

[M] L. J. Mordell, The congruence $((p-1) / 2) ! \equiv \pm 1(\bmod p)$, Amer. Math. Monthly 68 (1961), 145-146.

[PS] H. Pan and Z. W. Sun, New identities involving Bernoulli and Euler polynomials, J. Combin. Theory Ser. A 113 (2006), 156-175.

[S02] Z. W. Sun, On the sum $\sum_{k \equiv r(\bmod m)}\left(\begin{array}{l}n \\ k\end{array}\right)$ and related congruences, Israel J. Math. 128 (2002), 135-156.

[S03] - Combinatorial identities in dual sequences, European J. Combin. 24 (2003), 709-718.

[S06] - Polynomial extension of Fleck's congruence, Acta Arith. 122 (2006), 91-100.

[SD] Z. W. Sun and D. M. Davis, Combinatorial congruences modulo prime powers, Trans. Amer. Math. Soc., in press; http://arxiv.org/abs/math.NT/0508087.

[SP] Z. W. Sun and H. Pan, Identities concerning Bernoulli and Euler polynomials, Acta Arith. 125 (2006), 21-39.

[SW] Z. W. Sun and D. Wan, Lucas-type congruences for cyclotomic $\psi$-coefficients, Int. J. Number Theory, in press; http://arxiv.org/abs/math.NT/0512012.

[W] D. Wan, Combinatorial congruences and $\psi$-operators, Finite Fields Appl. 12 (2006), 693-703.

[Wa] L. C. Washington, Introduction to Cyclotomic Fields, 2nd ed., Grad. Texts in Math. 83, Springer, New York, 1997.

[We] C. S. Weisman, Some congruences for binomial coefficients, Michigan Math. J. 24 (1977), 141-151.

Department of Mathematics

Nanjing University

Nanjing 210093, People's Republic of China

E-mail: zwsun@nju.edu.cn

http://math.nju.edu.cn/ ${ }^{\sim}$ zwsun
Department of Mathematics University of California

Irvine, CA 92697-3875, U.S.A.

E-mail: dwan@math.uci.edu http://www.math.uci.edu/ dwan

Received on 27.6.2006

and in revised form on 17.1.2007 